\title{
Peripheral Autonomic Signals as Access Pathways for Individuals with Severe Disabilities: A Literature Appraisal
}

\author{
Stefanie Blain, Tom Chau ${ }^{*}$ and Alex Mihailidis
}

\author{
Bloorview Research Institute, Graduate Department of Rehabilitation Sciences, Institute of Biomaterials and Biomedical \\ Engineering, University of Toronto, Canada
}

\begin{abstract}
Many individuals with severe and multiple disabilities do not have an access pathway that enables them to interface with their environment because they are not afforded a binary switch that they can reliably control. While recent research has focused on the self-regulation of central signals of the autonomic nervous system (ANS) to create braincomputer interfaces (BCIs) for these individuals, there has been less focus on the peripheral signals of the ANS as an access pathway. An appraisal of the literature in the areas of biofeedback, polygraphy and mental exercises uncovered considerable evidence that peripheral ANS signals can be voluntarily controlled and thus have the potential to be used as an access pathway by the target population. However, the issues of speed, metabolic noise and pathological change must be addressed before peripheral ANS signals can be used as either a complementary or alternative access pathway to existing brain-computer interfaces.
\end{abstract}

Keywords: Autonomic nervous system, brain-computer interface, biofeedback, polygraphy, mental exercises.

\section{INTRODUCTION}

\subsection{Current Access Pathways to Assistive Technologies}

Able-bodied individuals who wish to express a functional intent conventionally use the modalities of speech or gestures as their preferred access pathway to interact with their environment. However, for individuals with severe motor impairments due for example to cerebral palsy, amyotrophic lateral sclerosis (ALS), brainstem stroke or spinal cord injury (herein referred to as the target population), these conventional access pathways are often not under voluntary control [1]. These conditions affect over half a million people worldwide, and have pathologies that cause deterioration of the mechanisms enabling somatic muscle control [2]. In these situations, assistive technologies such as augmentative and alternative communication (AAC), or environmental control system (ECS) technologies are often used to facilitate the individual's communication and interaction with his or her environment [3-5].

Both AAC and ECS assistive technologies can be operated, provided that the user has reliable control of a binary switch. As illustrated by the top arrow in Fig. (1), this binary switch is traditionally controlled via the muscles of the somatic nervous system. A wide variety of mechanical switches have been developed to exploit various types of controlled motor activities, including for example, head movement [6], eye blinks [7], eyelid movements [8], chin movements [9], muscle contractions via electromyographic (EMG) switches [10] and changes in gaze direction as determined by videooculography

*Address correspondence to this author at the Bloorview Research Institute, Bloorview Kids Rehab, Toronto, M4G IR8 Canada;

E-mail: tom.chau@utoronto.ca
(VOG) or electrooculography (EOG) [11]. These aforementioned access pathways, or methods of interfacing with the target assistive technology, are compromised in individuals with severe motor disabilities, either because the pathology of their conditions cause the loss of all voluntary control of their somatic muscles, or because their reliable motor movements are confounded by factors such as involuntary movements and fatigue. In these situations, conventional and commercially available access pathways are ineffective, rendering the individual unable to express functional intent or to interact with his or her environment [12]. That these individual are not afforded a consistent, reliable means of expressing functional intent results in a significant decrease in their independence, dignity and overall quality of life [13].

When research in the fields such as seizure control and behaviour and cognition demonstrated that individuals could be trained to voluntarily regulate their brain activity [14-16], the central autonomic pathway (Fig. 1, middle arrow) was pursued as an alternative to somatic control. To date, a number of brain signal components have demonstrated the ability to be self regulated, including electroencephalographic (EEG) components such as visual alpha rhythms, slow cortical potentials (SCP) [17] and sensorimotor rhythms (SMRs) [18, 19], neuronal firing as recorded by invasively implanted electrode arrays [20-22], and signals measured via magnetoencephlography (MEG), positron emission tomography (PET), functional magnetic resonance imaging (fMRI) [23,24] and near-infrared spectroscopy (NIRS) [25, 26]. Many of these signals have been harnessed to create a brain-controlled access pathway for the individuals of the target population, often referred to as brain-computer interfaces (BCIs) or brainmachine interfaces (BMIs). While central ANS BCI research has progressed to the point where clinical trials are viable [2729], it remains yet to be established whether or not the peripheral signals of the ANS have the potential to be a practical access pathway for individuals in the target population (bottom arrow of Fig. 1). In particular, the operant 
USER

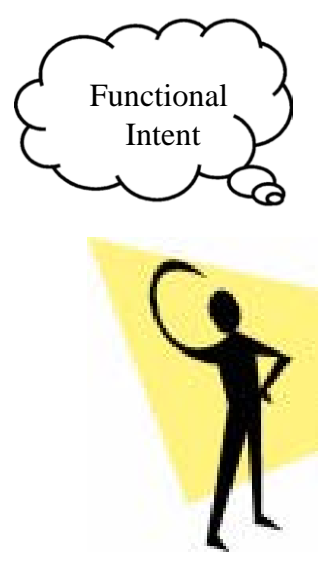

ACCESS PATHWAY

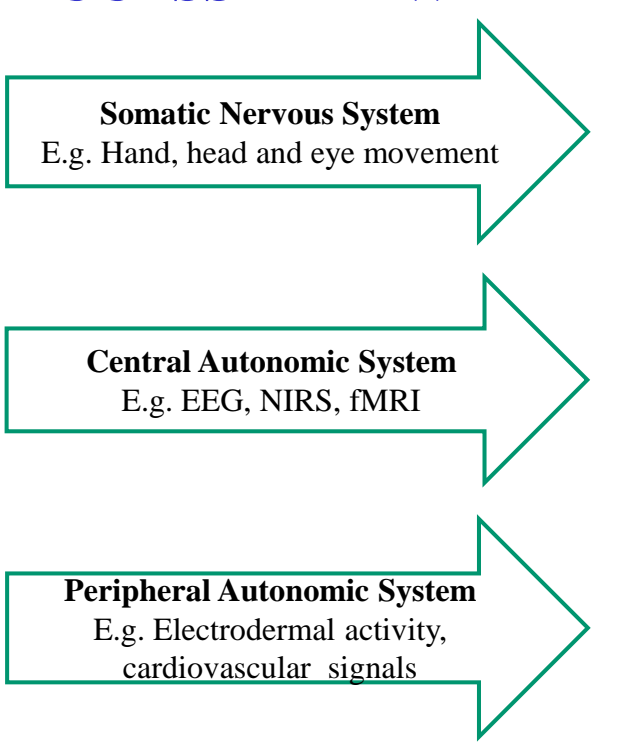

\section{ASSISTIVE TECHNOLOGY}

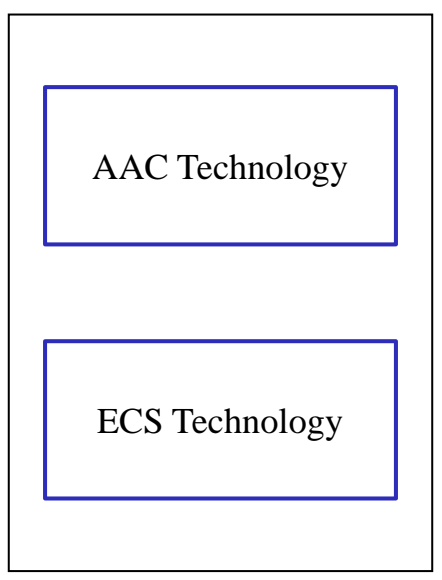

Fig. (1). Potential access pathways for the translation of functional intent into the control of assistive technologies $($ AAC $=$ augmentative and alternative communication; ECS = environmental control system).

conditioning and thus, voluntary control of peripheral ANS signals is still debated [30-32]. In addition to this uncertainty, utilizing peripheral ANS signals as an access pathway present several unique challenges, including a very slow rate of response (e.g. heart rate, $30 \mathrm{~s}$ per trial [33]), high metabolic noise and high incidence of pathological change in the target population [34]. While some peripheral autonomic signals have been investigated as access pathways [35-38], the question of learned control of these signals has yet to be answered and these aforementioned challenges remain unaddressed.

This paper surveys the relevant literature on operant control of selected peripheral autonomic signals as laid out in Fig. (2). We first present an overview of four autonomic signals of interest: electrodermal activity, heart rate, respiration rate and skin temperature. Subsequently, we appraise the evidence relating to voluntary control of these autonomic signals from three bodies of literature, namely, biofeedback, polygraphy and mental exercises. In light of the additional challenges of forging an access pathway from these signals, we conclude with a discussion of the issues of lengthy response time, metabolic noise and pathological signal change, and how these challengsses may be addressed in the context of a peripheral ANS access pathway.

\section{OVERVIEW OF AUTONOMIC SIGNALS}

\subsection{Electrodermal Activity}

Electrodermal activity (EDA), also commonly known as galvanic skin resistance (GSR), reflects sympathetic cholinergic function that induces changes in the skin's resistance to electrical conduction. It can be spontaneously or

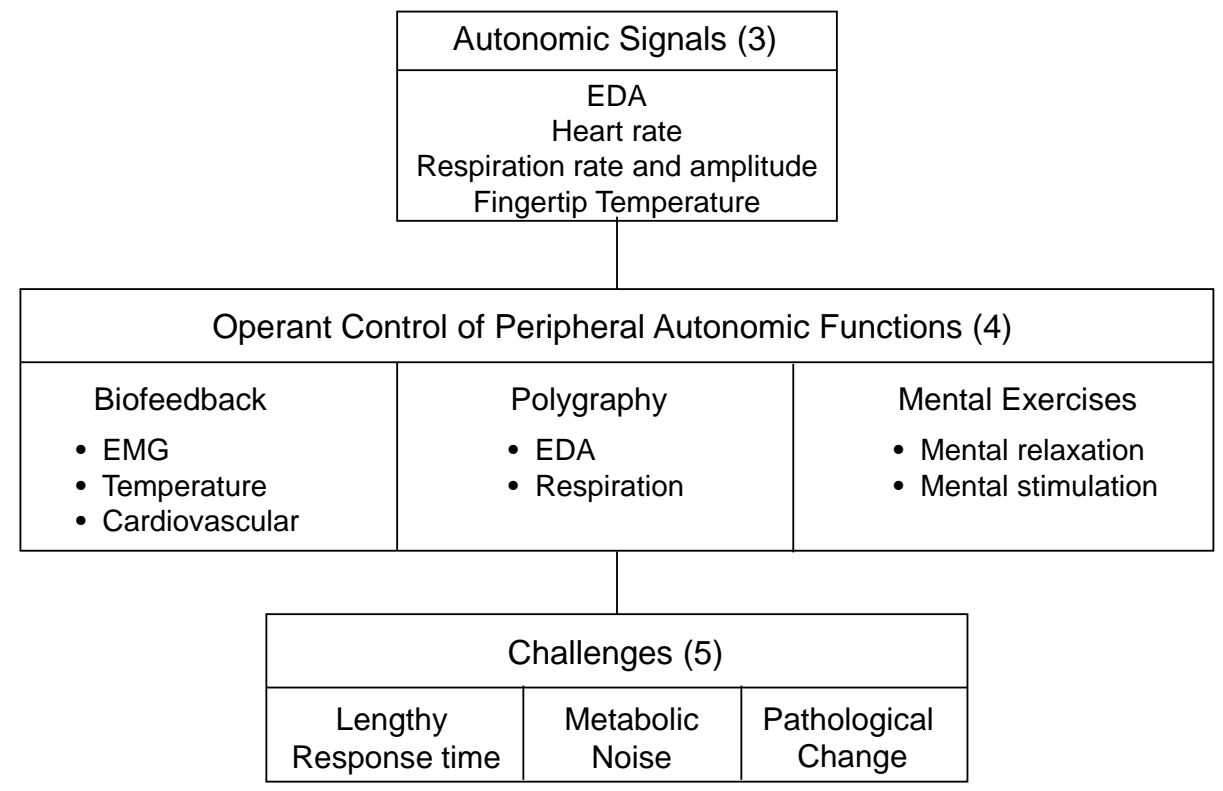

Fig. (2). The layout of the present review. Numbers indicate the corresponding sections of the paper. 
reflexively evoked by a variety of internal or externally applied arousal stimuli [39], and has been established over decades of research to be one of the most popular and convenient measures of ANS arousal [40]. In a crosssection of the human skin, each of the components of the 3-layered organ has constant resistive and capacitive properties, with the exception of the sweat glands. Innervated by the sympathetic branch of the autonomic nervous system, the sweat glands fill with an electrolytic fluid upon cholinergic stimulation, thereby changing the skin's overall electrical conductance. As such, measuring changes in the skin's resistive properties yields a sensitive measure of the level and extent of an individual's sympathetic activity [41]. Various arousal stimuli induce responses that initiate changes in EDA, including activation of the amygdala as a thermoregulatory response to startling and threatening stimuli [42] or in response to emotional or affective processes [40]; activation of the hippocampus in response to memory recall [43]; activation of the reticular formation and limbic structures as an orienting response to novel stimulus [44]; and activation from the basal ganglia due to an attention orienting reflex [40]. These stimuli result in an increase in electrodermal activity of more than $0.05 \mu \mathrm{S}$ within a 10 second interval a characteristic change known as an electrodermal reaction (EDR) [40]. These EDRs are superimposed on a slowlyevolving baseline EDA level, whose changes are a result of homeostatic and thermoregulatory processes, and not indicative of ANS activation [45].

EDA measurement is based on the principle of Ohm's law, $V=I R$, where $\mathrm{V}$, I and $\mathrm{R}$ represent voltage, current and resistance of the system, respectively. To measure R, the resistance of the skin, changes in current and/or voltage are recorded and related to changes in skin resistance via Ohm's law. Resistance is recorded via two electrodes affixed to the surface of a region of skin containing a high density of sweat glands, such as the palms of the hand and the soles of the feet. Many variations of EDA recording techniques have been established, as summarized in Fig. (3). The most well-established method of EDA recording - direct current measurement with a constant voltage source, is highlighted.

\subsection{Heart Rate}

Both sympathetic and parasympathetic branches of the autonomic nervous system project to nearly every region of the heart; their antagonistic effects enable the heart to respond to the rapidly changing metabolic requirements of the body. The dual innervation of the sinoatrial node enables heart rate to respond to arousal stimuli, yet makes it difficult to determine the precise patterns of heart rate change to stimuli of a specific valence, as these changes are highly stimulus dependent. Antagonistic effects are often observed between pairs of opposing stimuli, for example, heart rate undergoes deceleration when an individual's attention is focused on gathering sensory information from the environment, and accelerates during introspection and other incidences of dampened sensory input [46]. Heart rate has also been strongly correlated with emotional state, decelerating in response to disgust, increasing to a greater degree in negative than in positive emotions, and within negative emotions, increasing to a greater degree in response to sadness in comparison to anger [42].

Heart rate is commonly measured by logging the time interval between corresponding points of two successive cardiac cycles, as recorded by techniques such as the traditional electrocardiogram (ECG), a blood pressure cuff inflated to a pressure between $40-50 \mathrm{mmHg}$ [47], and photoplethysmography [48].

\subsection{Respiration Rate and Amplitude}

The body's control of respiratory musculature is unique, as it is regulated by both autonomic and somatic mechanisms. Somatic control dominates during periods of voluntary inspiration and expiration, such as speech and breath holding, and rhythmic autonomic signals control the diaphragm and intercostals muscles at all other times [49]. For some individuals with severe motor disabilities, voluntary control of respiration is not possible, and respiration rate is solely

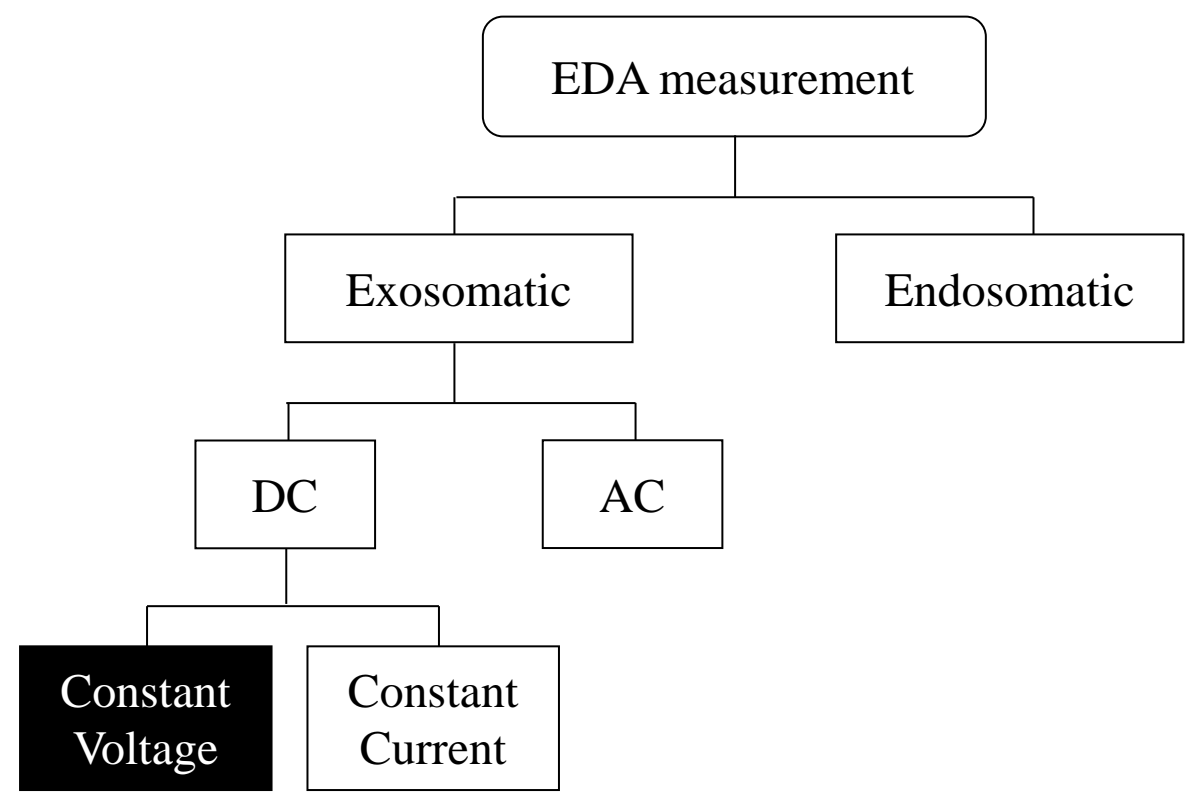

Fig. (3). The categories of potential EDA recording techniques. The most popular measurement technique in the literature is highlighted. 
regulated by the ANS. Heywood et al. studied the breathing patterns of an individual with locked-in syndrome, and results indicated that chemical inputs, such as the level of $\mathrm{CO}_{2}$ in the blood, are integrated in the medulla and cause autonomic adjustments in respiration rate and amplitude to maintain a normal end-tidal $\mathrm{P}_{\mathrm{CO} 2}$ of 39 - $40 \mathrm{mmHg}$ [50]. As arousal stimuli may cause metabolic changes that vary the level of $\mathrm{CO}_{2}$ in the blood, respiration rate and amplitude involuntarily increase during stress and decrease during relaxation.

Respiration rate and amplitude information are commonly calculated from basic algorithms applied to the respiratory cycle signals, as recorded via techniques such as a piezoelectric belt positioned around the thoracic area, translating the expansion and contraction of the lung cavity into changes in voltage [51], and low-inertia thermistors attached at the entrance of the nostril, using the detected warmer air exhaled during each respiratory cycle to reconstruct the pattern of respiration [52].

\subsection{Fingertip Temperature}

Fingertip temperature is a combination of long-term baseline changes that are caused by homeostatic thermoregulartory vasodilation and vasocontriction of blood vessels in the fingers, and transient changes in cutaneous microcirculation. Among these cutaneous vascular structures are arteriovenous anastomoses (AVAs), which are densely innervated by sympathetic nerve fibers [53]. Their response to sympathetic stimulation is dependent on the overall body temperature; in subjects whose finger temperature is above $33 \pm 2{ }^{\circ} \mathrm{C}$, sympathetic stimulation induces vasoconstriction, whereas below this temperature, the basic sympathetic vasoconstrictor tone is high and a vasoconstrictor response is not physiologically possible, consequently, the same stimulus will induce a vasodilator response [54]. Both vasoconstrictor and vasodilator responses occur after a latency period of approximately 15 seconds, and have durations of 20 to 40 seconds in length [55]. These microcirculatory responses have been recorded in response to arousal stimuli such as forced arithmetic, deep inspirations, sudden noises, and pain $[56,55]$, and are an established indicator of the state of an individual's ANS [54].

The minute changes in fingertip temperature are measured by attaching a thermistor to the finger to directly measure temperature change [55], or indirectly through infrared thermography [57], which detects the changes in fingertip infrared emissions over time.

A summary of the physiological origins, stimulus mechanisms and measurement techniques for each autonomic signal discussed in this section is presented in Table $\mathbf{1 .}$

\section{OPERANT CONTROL OF PERIPHERAL AUTO- NOMIC FUNCTIONS}

Each of the four autonomic physiological signals discussed in Section 3 demonstrates a specific, reproducible response to sympathetic stimuli. We now proceed to appraise the literature wherein an individual intentionally and intrinsically generates these arousal stimuli, bringing these autonomic responses under conscious control. Preliminary reviews of the literature identified three areas wherein a number of techniques are used to regulate both individual and combinations of autonomic signals: biofeedback, polygraphy and mental exercises. A subsequent, extensive literature review was conducted in Ovid Medline, Scholars Portal and PubMed, combining the key word autonomic nervous system with the keywords biofeedback and polygraphy OR lie detection in separate searches. The literature review for mental exercises was subdivided into three searches: autonomic nervous system AND (1) mental arithmetic, (2) meditation, and (3) motor imagery. The search was limited to English communications dating onwards of 1960 and returned 416 references. Subsequently, articles were included if they examined the effect of biofeedback, polygraphy or mental exercise on the autonomic nervous system using either: a) a control group that did not undertake the activities used to generated ANS changes; or b) a pre-post study design, where the ANS signals

Table 1. Overview of Autonomic Signals

\begin{tabular}{|c|c|c|c|}
\hline Autonomic Signal & Physiological Origin & Typical Mechanisms of Modulation & Measurement Technique \\
\hline Electrodermal Activity & $\begin{array}{l}\text { Sympathetic innervation of sweat } \\
\text { glands }\end{array}$ & $\begin{array}{l}\text { - Emotional thoughts } \\
\text { - Orienting response } \\
\text { - Startle response } \\
\text { - Memory recall }\end{array}$ & $\begin{array}{l}\text { - } \mathrm{Ag} / \mathrm{Ag}-\mathrm{Cl} \text { electrodes on fingers or } \\
\text { soles of foot }\end{array}$ \\
\hline Heart Rate & $\begin{array}{l}\text { Sympathetic and parasympathetic } \\
\text { innervation of the sino-atrial node }\end{array}$ & $\begin{array}{l}\text { - Internal vs. external attention focus } \\
\text { - Emotional state }\end{array}$ & $\begin{array}{l}\text { - EKG } \\
\text { - Blood pressure cuff } \\
\text { - Photoplethysmography }\end{array}$ \\
\hline Respiration Rate & $\begin{array}{l}\text { Sympathetic innervation of the } \\
\text { diaphragm and intercostals muscles }\end{array}$ & $\begin{array}{l}\text { - Metabolic change } \\
\text { - Emotional state }\end{array}$ & $\begin{array}{l}\text { - Piezoelectric belt } \\
\text { - Low-inertia thermistor }\end{array}$ \\
\hline Skin Temperature & $\begin{array}{l}\text { Sympathetic innervation of the } \\
\text { arteriovenous anastomoses }\end{array}$ & $\begin{array}{l}\text { - Emotional state } \\
\text { - Forced arithmetic } \\
\text { - Deep inspirations } \\
\text { - Startle and pain }\end{array}$ & $\begin{array}{l}\text { - Thermistor } \\
\text { - Infrared thermography }\end{array}$ \\
\hline
\end{tabular}


of an individual were measured prior to and following the intervention. According to these criteria, 31 articles were retained and included in the appraisal. The evidence gathered from this body of literature pertaining to the conscious control of peripheral ANS signals is presented in the following sections.

\subsection{Biofeedback}

Early experiments conducted by Miller, Kimmel and Engel demonstrated operant conditioning of several autonomic signals, and gave rise to the field of biofeedback [32, 58, 59]. In biofeedback, physiological monitoring devices are used to provide individuals with information on the state of one or more of their physiological signals, and individuals are trained to use this feedback to learn to voluntarily regulate these signals. While many instances involving biofeedback training did not withstand clinical trial and rigorous scientific testing, there nevertheless remains a relatively small, but scientifically rigorous body of work that clearly demonstrates that control over an autonomic signal can be attained if an individual receives feedback about the state of a signal as it changes over time [60]. The physiological signals that are predominantly used as feedback can be classified into three general groups: (1) electromyography (EMG); (2) temperature; and (3) cardiovascular.

\subsubsection{Electromyographic Biofeedback}

Electromyographic signals are generated from the electric activity regulating muscle contractions. Studies have demonstrated that while individuals with late-stage amyotrophic lateral sclerosis and motor neurone disease are unable to generate overt physical movement, they remain able to generate sufficient myoelectric activity to be detected by commercial EMG sensors [10]. In EMG biofeedback, displaying the EMG signal enables an individual to learn to regulate this activity, and correspondingly, to regulate muscle tension. A number of pathological states result from either insufficient or excess tension in both somatically and autonomically innervated muscles; EMG biofeedback treatments have been successfully used to teach the individual to consciously regulate these pathological muscles. Its power as a technique to teach individuals to consciously regulate involuntary muscle activity has been illustrated through well-controlled studies. Flor and Birbaumer [61] randomly assigned 57 patients with chronic back pain and 21 patients with temporomandibular pain to EMG biofeedback, cognitive-behavioural therapy or conservative medicine treatment groups. The biofeedback group received EMG biofeedback from the site of the pain in the form of an auditory tone that stopped when they had succeeding in relaxing the site muscles to a pre-defined threshold. While all three groups improved post-treatment, the biofeedback group demonstrated the most substantial reduction in pain severity. Other studies have also illustrated the ability for individuals to gain mental control over involuntary physiological functions by using EMG biofeedback to develop control over the muscles responsible for voiding disorders. Engel's treatment of incontinence fed back information on rectal distension to patients, training them to learn external sphincter control within four treatment sessions [62]. Khen-Dunlop et al. used similar EMG biofeedback techniques to treat bladder over-activity. In a prepost study design, 60 children with voiding disorders received visual biofeedback of their perineal muscles, training over 10 weekly sessions to learn to voluntarily control the tension of the muscle. Six months after the last session, $96 \%$ of the children with diurnal incontinence and $83 \%$ of the children with nocturnal incontinence were cured or experienced a reduction to at least half of their previous number of wet episodes per week [63].

\subsubsection{Temperature Biofeedback}

Temperature biofeedback has most often been investigated as a treatment for Raynaud's phenomena, a painful manifestation of digital artery vasospasm that is typically provoked by cold exposure [64]. These biofeedback studies can be categorized into those conducted with able-bodied individuals, and those conducted with individuals with Raynaud's phenomena. Able-bodied studies have rigorously demonstrated that providing feedback of fingertip temperature enables individuals to learn to voluntarily increase digital blood flow, which reliably increases their skin temperature. Furthermore, once this behaviour has been learned, the response can be reliably generated without feedback, and in different temperature environments [65-67]. Studies with individuals with Raynaud's phenomena also provide evidence that fingertip temperature can be statistically significantly changed at will after temperature biofeedback training, and that once learned, temperature control can be generated without feedback, generalized to locations outside the laboratory, and retained over time [64]. While the results of a recent large $(n=313)$ multi-center trial demonstrated that temperature biofeedback was inferior to sustained-release nifedipine for treating primary Raynaud's phenomena, these results simply call into question the clinical significance of biofeedback training as a treatment for this condition, and does not diminish the statistical significance of the results that demonstrate the ability for a physiological function to be voluntarily controlled [64].

\subsubsection{Cardiovascular Biofeedback}

Within biofeedback research, cardiovascular biofeedback has generated substantial interest as a potential non-invasive treatment of serious cardiovascular diseases. Kristt and Engel [68] demonstrated that blood pressure could be brought under voluntary control in the laboratory and surrounding environments using operant conditioning techniques. Five patients with high blood pressure of unknown origin were trained to both raise and lower their blood pressure by monitoring a display of lights that corresponded to their current blood pressure level and the direction of the change that was required. Four of the five subjects were able to evoke statistically significant changes in their blood pressure in the required direction after approximately 50 sessions. Using identical training techniques, Bleecker and Engel [58] were also able to demonstrate that able-bodied men and subjects with atrial fibrillation were able to learn to slow, speed, or cyclically slow and speed their heart rate.

In the three decades since Engel's initial studies, studies in the field of cardiovascular biofeedback have mainly focused on whether this technique is effective in the treatment of cardiovascular diseases such as hypertension. The current 
consensus of medical opinion does not favour the routine application of relaxation and biofeedback to treat hypertension [69]. Nevertheless, studies have demonstrated that consistent antihypertensive effects have been achieved by biofeedback, especially when the therapy includes a respiratory retraining and a slow breathing component. Aivazyan et al. demonstrated that subjects trained with a combination of relaxation strategies such as autogenic training, biofeedback, and breathing were able to significantly decrease both their systolic and diastolic blood pressure and vascular resistance in comparison to a control group that was simply instructed to relax [70]. Similarly, in a controlled trial, Patel and North compared a group of subjects who were taught structured relaxation involving yoga and biofeedback to a group that practiced uninstructed relaxation and found significantly greater decreases in blood pressure in the treatment group [71]. A recent meta-analytic review of all biofeedback methods of treating high blood pressure found an average decline of $6.7 / 4.8 \mathrm{mmHg}$ for systolic and diastolic blood pressure respectively, a significantly greater reduction in comparison to inactive control treatments [72]. As in the situation of temperature biofeedback treatment of Raynaud's phenomena, there is ongoing debate on the clinical significance of the results achieved by cardiovascular biofeedback $[60,69,73,74]$.

All three of the aforementioned types of biofeedback have been effective in treating migraine. In a recent metaanalysis by Nestoriuc and Martin [75], a review of 55 randomized controlled trials and pre-post trials demonstrated a medium effect size for all three modes of biofeedback intervention for treating migraine. This effect size represents a consistent reduction in symptoms by more than half a standard deviation, which is remarkably high in the area of chronic pain. On average, these effects remained stable for over a year post-treatment, and reduced the frequency and duration of the migrane attacks more than the medication-intake.

\subsection{Polygraphy}

In the field of polygraphy, an individual's peripheral autonomic signals are monitored as he or she answers a series of questions with the objective of distinguishing truthful from deceptive responses. The Guilty Knowledge Test (GKT) is one such psychophysiological method for the detection of deception and has been extensively studied. In this test, individuals are instructed to respond 'no' to a series of multiple-choice questions, each with one option containing details that would be known to the perpetrator of a crime, but not to innocent suspects, and several control alternatives [76]. It is assumed that as the guilty individual deceptively responds 'no' to the relevant alternative, his or her autonomic signals will exhibit systematic differences in comparison to those generated as the individual truthfully responds to the control alterative. In a review of 10 GKT laboratory experiments, BenShakhar and Furedy [77] showed that across the studies, $83.9 \%$ of 248 guilty suspects and $94.2 \%$ of 208 innocent suspects were correctly identified, although the validity of the GKT in real life polygraph records is lower [78]. One potential explanation for this discrepancy may be that in the real life situations, guilty suspects practiced countermeasures to avoid detection. These countermeasures are of interest, as they illustrate that subtle physical and mental techniques can invoke deliberate changes in the patterns of an individual's peripheral ANS response patterns. Physical countermeasures involve practices such as the self-inflection of pain or heavy breathing after a control question to stimulate an ANS response. Mental countermeasures involve practices such as mental relaxation after the presentation of a relevant question to suppress an ANS response, and mental stimulation after the presentation of a control question to deliberately generate an ANS response; the effect of these mental techniques will be the focus of this section.

\subsubsection{Electrodermal Activity in Polygraph Research}

Electrodermal activity is the most popular discriminatory signal for the GKT. Electrodermal reactions (EDRs) are generated in response to recognition events, even when subjects report not consciously attending to the stimulus [40, 79], and as such, would be generated when a guilty subject is presented the details of his or her crime, but would be absent when an innocent subject is presented identical details. Most polygraph studies before 1990 used EDA as the sole dependent variable and were nonetheless able to obtain high accuracy estimates of deception [80, 81]. The generation of EDRs in response to recognition has been replicated over a variety of conditions, including motivation to deceive, perceived accuracy of polygraphy, free or forced choice of deceptive items, mode of response (e.g., verbal or pressing keys) and degree of emotional involvement [82]. Mental countermeasures against this response involve either the suppression of an EDR after a recognition event, or the voluntary generation of an EDR after a control question. In addition, this voluntary control must be exerted within less than ten seconds in order to successfully suppress or realistically generate an EDR. To determine the effectiveness of these countermeasures, Elaad and Ben-Shakhar [47] investigated the effect on EDA of invoking relaxing thoughts that dissociate the subject mentally both after the presentation of a relevant question, and continually throughout the GKT. Their results demonstrate that EDRs can be voluntarily repressed via mental relaxation techniques, as the detection of guilty subjects was significantly higher in the group that did not practice countermeasures. Ben-Shakhar and Dolev [51] investigated the opposite countermeasure strategy - generating an excitatory ANS response to a control question. In a randomized controlled trial $(n=129)$, innocent individuals and individuals who committed a mock crime underwent a GKT polygraph test. The individuals who committed the mock-crime were subdivided into 3 groups, each of which was tested under a different condition: group 1 received no countermeasure instruction, groups 2 and 3 were instructed to attempt to deceive the examiner by recalling an emotional situation to generate a response to irrelevant alternatives, and group 3 was given the opportunity to rehearse using this mental countermeasure on a polygraph machine prior to the test. Results confirmed that individuals equipped with the countermeasure strategy were indeed able to voluntarily generate EDRs, thereby significantly lowering detection rates. Furthermore, the amount of prior practice of this countermeasure was not a predictor of an individual's ability to voluntarily generate EDRs. 


\subsubsection{Respiration in Polygraph Research}

The respiration length line (RLL), a combination of both respiration amplitude and respiration cycle time, provides a global score of respiration suppression and is a component of the orienting response [83]. Respiration suppression occurs during the act of deception, and consequently, RLL can be used to accurately differentiate between a guilty and innocent subject [84] and follows EDR amplitude as the second most informative measure in detecting deception [85]. In a mock-crime GKT experiment carried out on 270 guilty subjects, Timm [86] reported that RLL was more effective than EDRs in detecting guilty knowledge. In addition, using a combination of EDA measures and RLL provided higher detection accuracy than either measure did individually.

Intuitively, individuals are more likely to be able to voluntarily control their respiration suppression response in comparison to other physiological signals (e.g., EDA), as respiratory musculature is also innervated by somatic mechanisms. However, Ben-Shakhar and Dolev [51] demonstrated that while EDA is affected by mental countermeasures, there are no significant differences in RLL measures between guilty subjects who do and do not practice mental countermeasures. These results indicate that mental activities do not affect respiration patterns; other forms of control are required to generate voluntary changes in this physiological signal.

\subsection{Mental Exercises}

This section further explores other mental techniques used to both suppress and generate consistent changes in an individual's physiological responses by surveying the literature in the areas of meditation, mental arithmetic and mental imagery.

\subsubsection{Mental Relaxation and Meditation}

Meditation refers to a large variety of mental practices that result in voluntary changes in the state and contents of consciousness [87]. While it has been a constituent of major religions such as Hinduism and Buddhism for centuries, only recently have the physiological effects of this practice been investigated. During Transcendental Meditation (TM), the subject undergoes a state of transcending, an experience described as "taking the mind from the experience of a thought to finer states of the thought" [88], and its practice has been linked in basic and clinical research to decreasing anxiety, hypertension, arthrosclerosis and substance abuse [89]. In an investigation of the changes in autonomic patterns during the state of transcending, autonomic signals were recorded from 30 participants who had been using the TM technique for an average of 5.4 years. Results demonstrated a statistically significant difference in EDA and respiratory patterns during periods of transcending compared to other states of consciousness. Similar results have been achieved in individuals without formal meditation training. Kaushik et al. studied the ANS patterns of 100 patients with essential hypertension during baseline states and states of mental relaxation. Subjects were asked to lie in a comfortable position and undergo complete mental relaxation by thinking of some pleasant thought for 10 minutes. By practicing these mental relaxation techniques, subjects experienced a significant drop in systolic blood pressure and respiration rate, and a significant increase in fingertip temperature. The investigators concluded that mental relaxation was an effective technique to reduce blood pressure in hypertensive patients, and could also induce significant changes in an individual's respiration patterns and peripheral skin temperature [90].

\subsubsection{Mental Stimulation}

Tasks requiring mental arithmetic are used frequently in research to generate states of mental stress and stimulation [91-93], and the effects of performing mental arithmetic on the components of the ANS are well studied. During silent arithmetic, individuals experience a significant increase in heart rate, electrodermal activity, the pre-ejection period of the cardiac cycle, cardiac output, and respiration rate, accompanied by a significant decrease in the stroke volume of the heart $[55,93]$. These changes are observed in the recorded signals within 40 seconds of initiating the mental task. Forced arithmetic also causes statistically significant and reproducible vasodilatory responses in cold subjects [56].

Another commonly used mental stimulation technique is motor imagery - a mental simulation of voluntary motor actions. During this mental exercise, the autonomic system responds in a similar manner to actual exercise, even though muscle movement does not actually occur [94]. When elite athletes perform motor imagery of their sport, significant increases are observed in skin conductance levels, heart rate and respiration rate, while skin blood flow, skin temperature and respiration amplitude undergo significant decreases [52, $95,96]$. The degree of these responses is proportional to the mental effort exerted by the subject. Through principle component analysis, Deschaumes-Molinaro et al. (1992) [96] determined that in the specific tasks of motor imagery of firearm and archery shooting, skin conductance, skin blood flow and skin temperature underwent the most significant changes in response to mental imagery. These studies demonstrate that both mental arithmetic and mental imagery are effective tasks that individuals can use to voluntarily generate changes in the patterns of their peripheral ANS signals.

\section{DISCUSSION}

The amassed literature suggests that peripheral autonomic signals have the ability to be voluntarily controlled through a variety of mental techniques, and thus, have the potential to become an access pathway for individuals with severe and multiple disabilities. However, the clinical viability of these pathways hinges upon the resolution of at least three significant challenges: 1) the speed of peripheral signals in comparison to central signals; 2) the metabolic noise in peripheral pathways; and 3) the incidence of pathological change in individuals of the target population. Each of these challenges is discussed below.

\subsection{Challenge 1 - Slow Rate of Response}

To date, the majority of access channels for those who lack the physical ability to interact with their environment have focused on the central autonomic signals from the brain, and the corresponding developments in the area of brain computer 
interfaces have been plentiful and impressive [19, 30, 34 , 97]. The enormous advantage of these central signals lies in the fact that their maximum achievable speed is far greater than what can be achieved with the peripheral autonomic signals. While some EEG-based BCIs have achieved transfer rates of up to 27.15 bits/minute [98], autonomic responses can be reliably detected perhaps once every 30 seconds. For individuals with terminal conditions such as amyotrophic lateral sclerosis (ALS), it is conceivable that the speed and accuracy of their access channel are of utmost importance, forgoing all other considerations. However, for individuals with more stable conditions, this is not necessarily the case. Testimonies from individuals who rely on access technologies indicate that the speed of access may not be the all-pervasive top priority, at the expense of all other consideration. In a survey of 17 ALS patients who were extensively informed of the advantages (e.g., greater speed) of a surgically implanted electrode-based BCI over a non-invasive BCI, 16 refused the procedure in favour of the slower and more error-prone non-invasive device, arguing that when one was completely paralyzed, time was not an issue [97]. The comments of a father of one of the participants in the Cyberlink research, a brain-body interface for individuals with severe TBI, further illustrates this de-emphasis of temporal urgency.

Even one definite yes or no response to a question from a head-injured son performed just once a week is a joyful event that no person who is not in this situation can appreciate or fully understand. For him, it fully justifies the trouble of setting up the hardware and software [99].

It is conceivable that some individuals in the target population and their caregivers may place a greater priority on factors such as the convenience of instrumentation and aesthetics of their access pathway over its maximum achievable speed $[100,101]$. While not suited to all individuals in the target population, a peripheral autonomic signal based access pathways may provide an alternative to the faster central autonomic signal based access pathways, enabling the user to choose the technology that best suits his or her personal needs and lifestyle [102].

\subsection{Challenge 2 - Metabolic Noise}

The primary function of the autonomic system is to maintain homeostasis in the body. Consequently, voluntarily generated changes in the signals of this system will be confounded by a significant amount of metabolic noise, as the body responds involuntarily to environmental stimuli. However, this level of noise does not entirely eliminate the possibility of using peripheral autonomic signals as an access pathway - the pathway is still viable provided that an intelligent system can recognize and discriminate between volitional activity and variations due to instinctive reactions and the maintenance of homeostasis. Several methods can be employed for this purpose. One possibility is to extract information from the combination of various physiological signals. For example, deep inspirations function as sympathetic stimuli, and cause an electrodermal reaction [103]. If electrodermal activity is employed as an access pathway, knowledge of this respiratory-electrodermal interaction can be used to discriminate between voluntarily generated EDRs, and EDRs that are a result of metabolic processes such as deep breathing or coughing. This so called source-separation technique has been successfully employed to improve the classification accuracy of an EDA-based access pathway; filtering respiration patterns from the raw EDA signal improve one subject's classification accuracy of resting and excited mental states [35]. Further filtering of the electrodermal signal for metabolic noise can be accomplished by examining the cardiac patterns of an individual. Studies in fields such as emotion recognition illustrate that a startle stimulus elicits both an electrodermal reaction and cardiac acceleration [104], while activities such as mental arithmetic elicit an electrodermal reaction and cardiac deceleration [94]. By tracking cardiac acceleration and deceleration patterns, an intelligent system could potentially discriminate between involuntary and voluntarily generated EDRs, eliminating activations that occur as an involuntary response to startling stimuli. Further work must be done to establish the efficacy of this source-separation approach, summarized in Fig. (4), to isolate self-regulated change from metabolic noise in peripheral autonomic signals specifically for the purpose of access to the environment or communication.

\subsection{Challenge 3- Pathological Change}

Physiological signals vary significantly between individuals, and may undergo further changes in the presence of various pathologies. To create a meaningful access pathway, it is crucial to conduct individual baseline recordings to determine which physiological signals are labile, and therefore potentially usefully for decoding an individual's mental state. For example, individuals with late-stage ALS must be artificially ventilated to survive [105]; clearly,

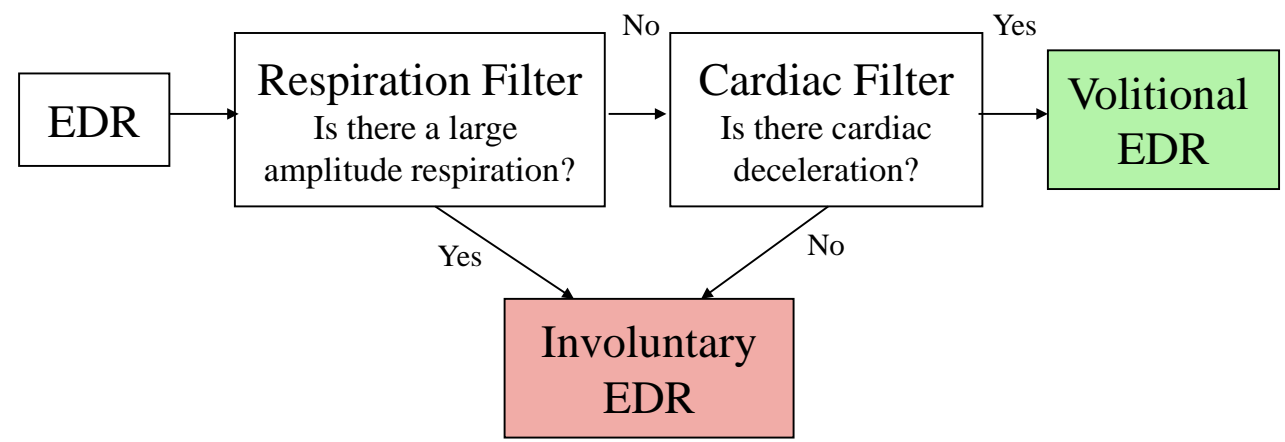

Fig. (4). A potential method of accounting for metabolic noise in peripheral autonomic signals. Large amplitude respirations and cardiac accelerations are indicative of electrodermal reactions (EDRs) generated from external stimuli - these can be used as part of a filter to discriminate voluntary from involuntary changes in an individual's ANS. 
respiration patterns yield no meaningful information about the individual. Similarly, individuals with complete spinal cord injury demonstrate no changes in electrodermal activity below the level of injury [106]. An access channel based on physiological signals for these individuals cannot include this particular signal. Within the identified labile signals, it is likely that the signal characteristics vary from those of the able-bodied population. For example, the defining characteristics of electrodermal reactions latency and amplitude - are significantly different between individuals with multiple sclerosis (MS) in comparison to a control group of able-bodied participant [107]. In a study with 25 individuals with ALS, all exhibited the ability to produce electrodermal reactions in their hand, but the mean EDR latency was prolonged compared to that of the control group [108]. However, these pathological deviations from the typical response do not necessarily eliminate an access pathway from consideration. As long as the individual is able to produce a significant change away from his or her typical baseline pattern, the pathway may still be useful. For example, regardless of the baseline latency value of the EDR of an individual with ALS or $\mathrm{MS}$, if the individual is able to voluntarily increase or decrease the latency, this information can be used to create an access pathway tailored to his or her unique physiological patterns. Blain et al. (2007) illustrated benefits of using individually-tailored access pathways in a study with six able-bodied individuals. Using algorithms suited to each individual's physiological patterns to classify EDA, a subject's mental state could be classified as either resting or active to an accuracy of over $80 \%$, a higher accuracy than has been achieved in other studies with non-specific algorithms to classify electrodermal activity [35].

\section{CONCLUSION}

The appraised literature in the fields of biofeedback, polygraphy and mental exercises support the notion of conscious regulation of peripheral autonomic signals using mental activities. If the issues of slow response, metabolic noise and pathological change can be addressed, a peripheral autonomic signal-based access pathway may serve as an alternative or complementary source of information to central autonomic signal-based braincomputer interfaces. Further research to explore the potential and limitations of peripheral autonomic access pathways is warranted.

\section{REFERENCES}

[1] Fager S, Beukelman D, Karantounis R, et al. Use of safe-laser access technology to increase head movement in persons with severe motor impairment: A series of case reports. Augment Altern Comm 2006; 22(3): 222-9.

[2] Barnet HJM, Mohr JP, Stein BM, et al. Stroke: Patho-Physiology, Diagnosis \& Management, $2^{\text {nd }}$ ed. Churchill Livingstone 1992.

[3] Fried-Oken M, Fox L, Rau MT, et al. Purposes of AAC device use for persons with ALS as reported by caregivers. Augment Altern Comm 2006; 22(3): 209-21.

[4] Murphy J. Communication strategies of people with ALS and their partners. Amyotroph Lateral Scler Other Motor Neuron Disord 2004; 5: 121-6.
[5] Craig A, Tran Y, McIsaac P, et al. The efficacy and benefits of environmental control systems for the severely disabled. Med Sci Monit 2005; 11(1): 32-9.

[6] Evans DG, Drew R, Blenkhorn P. Controlling mouse pointer position using an infrared head-operated Joystick. IEEE T Rehabil Eng 2000; 8(1): 107-17.

[7] Lancioni GE, O'Reilly MF, Singh NN, et al. Children with multiple disabilities and minimal motor behavior using chin movements to operate microswitches to obtain environmental stimulation. Res Dev Disabil 2006; 27(3): 637-41.

[8] Lancioni GE, OReilly MF, Singh NN, et al. A new microswitch to enable a boy with minimal motor behavior to control environmental stimulation with eye blinks. Behav Intervent 2005; 20(2): 147-53.

[9] Lancioni GE, Singh NN, O'Reilly MF, Siagfoos J, Oliva D, Costantini A. An optic micro-switch for an eyelid response to foster environmental control in children with minimal motor behavior. Pediatr Rehabil 2006; 9(1): 53-6.

[10] Gryfe P, Kurtz I, Gutmann M, et al. Freedom through a single switch: coping and communicating with artificial ventilation. J Neurol Sci 1996; (139 Suppl): 132-3.

[11] Bates R, Istance H, Oosthuizen L, Majaranta P. Survey of De-Facto standards in eye tracking [Online]. 2007 Mar 21, [cited 2007]. Available from: http://www.cogain.org/results/reports/COGAIND2.1.pdf

[12] Wellings DJ, Unsworth J. Fortnightly review: Environmental control systems for people with a disability: an update. BMJ 1997; 315: 40912.

[13] Petry K, Maes B, Vlaskamp C. Domains of quality of life of people with profound multiple disabilities: the perspective of parents and direct support staff. J Appl Res Intellect 2005; 18: 35-46.

[14] Kamiya J. Biofeedback and self-control: An Aldine reader on the regulation of bodily processes and consciousness. Chicago, United States: Aldine 1971.

[15] Birbaumer N. Operant enhancement of EEG-theta activity: Aspiration and reality. In: Beatty J, Legewie H, Eds. Biofeedback and Behavior. New York: Plenum Press 1977; pp. 135-46.

[16] Sterman MB, Friar L. Suppression of seizures in an epileptic following sensorimotor EEG feedback training. Electroen Clin Neuro 1972; 33(1): 89-95.

[17] Birbaumer N, Ghanayim N, Hinterberger T, et al. A spelling device for the paralysed. Nature 1999; 398: 297-98.

[18] Pfurtscheller B, Neuper C, Muller GR, et al. Graz-BCI: State of the art and clinical applications. IEEE T Neur Sys Reh 2003; 11: 177-80.

[19] Wolpaw JR, Birbaumer N, McFarland DJ, et al. Brain-computer interfaces for communication and control. Clin Neurophysiol 2002; 113(6): 767-91.

[20] Kennedy PR, Bakay RAE, Moore MM, et al. Direct control of a computer from the human central nervous system. IEEE T Neur Sys Reh 2000; 8: 198-202.

[21] Serruya MD, Hatsopoulous NG, Paninski L, et al. Brain-machine interface: Instant neural control of a movement signal. Nature 2002; 416(6877): 141-2.

[22] Leuthardt EC, Schalk G, Wolpaw JR, et al. A brain-computer interface using electrocorticographic signals in humans. J Neural Eng 2004; 1 : 63-71.

[23] Hinterberger T, Weiskopf N, Veit R, et al. An EEG-driven braincomputer interface combined with functional magnetic resonance imaging (fMRI). IEEE T Bio-Med Eng 2004; 51: 971-4.

[24] Weiskopf N, Veit R, Erb M, et al. Physiological self-regulation of regional brain activity using real-time functional magnetic resonance imaging (fMRI): Methodology and exemplary data. Neuroimage 2003; 19: 577-86.

[25] Coyle S, Ward T, Markham C, et al. On the suitability of near infrared (NIR) systems for next-generation brain-computer interfaces. Physiol Meas 2004; 25: 815-22.

[26] Sitaram R, Zhang H, Guan C, et al. Temporal classification of multichannel near-infrared spectroscopy signals of motor imagery for developing a brain-computer interface. Neuroimage 2007; 34(4): 1416-27.

[27] Neuper C, Müller GR, Kübler A, et al. Clinical application of an EEG-based brain-computer interface: A case study in a patient with severe motor impairment. Clin Neurophysiol 2003; 114(3): 399-409.

[28] Sellers EW, Donchin E. A P300-based brain-computer interface: Initial tests by ALS patients. Clin Neurophysiol 2006; 117(3): 53848. 
[29] Birbaumer N, Kubler A, Ghanayim N, et al. The thought translation device (TTD) for completely paralyzed patients. IEEE T Rehabil Eng 2000; 8(2): 190-3.

[30] Birbaumer N. Breaking the silence: Brain-computer interfaces (BCI) for communication and motor control. Psychophysiology 2006; 43(6): 517-32.

[31] Dworkin BR. Learning and physiological regulation. Chicago, USA: University of Chicago Press 1993.

[32] Miller NE. Learning of visceral and glandular responses. Science 1969; 163(3866): 434-45.

[33] Williams RJ, Roberts LE. Relation of learned heart rate control to self-report in different task environments. Psychophysiology 1988; 25: 354-65.

[34] Kübler A, Kotchoubey B, Kaiser J, et al. Brain-Computer Communication: Unlocking the locked in. Psychol Bull 2001; 127(3): 358-75.

[35] Blain S, Mihailidis A, Chau T. Assessing the potential of electrodermal activity as an alternative access pathway. Med Eng Phys 2008; 30(4): 498-505.

[36] Moore M, Dua GA Galvanic Skin Response Interface for People with Severe Motor Disabilities: ASSETS; Atlanta, Georgia; October 18-20, 2004.

[37] Tsukahara R, Aoki H. Skin potential response in letter recognition task as an alternative communication channel for individuals with severe motor disability. Clin Neurophysiol 2002; 113(11): 1723-33.

[38] Wilhelm B, Jordan M, Birbaumer N. Communication in lockedin syndrome: effects of imagery on salivary $\mathrm{pH}$. Neurology 2006; 67(3): 534-5.

[39] Vetrugno R, Liguori R, Cortelli P, et al. Sympathetic skin response: Basic mechanisms and clinical applications. Clin Auton Res 2003; 13(4): 256-70.

[40] Boucsein W. Electrodermal activity. New York, USA: Plenum Press 1992.

[41] Fowles D. The eccrine system and electrodermal activity. In: Coles MGH, Donchin E, Porges SW, Eds. Psychophysiology New York: Guilford Press 1986; pp. 51-96.

[42] Edelberg R. Mechanisms of electrodermal adaptions for locomotion, manipulation, or defense. Prog Psychol Physiol 1973; 5: 155-209.

[43] Homma S, Matsunami K, Yan HX, Deguchi K. Hippocampus in relation to mental sweating response evoked by memory recall and mental calculation: a human electroencephalography study with dipole tracing. Neurosci Lett 2001; 305(1): 1-4.

[44] Venables PH, Christie MJ. Electrodermal Activity. In: Martin I, Venables PH, Eds. Techniques in Psychophysiology New York: John Wiley 1980; pp. 2-67.

[45] Hot P, Naveteur J, Leconte P, et al. Diurnal variations of tonic electrodermal activity. Int J Psychophysiol 1999; 33(3): 223-30.

[46] Lacey JI. Somatic response patterns and stress: some revisions of activation theory. In: Appley MH, Trumbull R, Eds. Psychological stress: issues in research New York: AppletonCentury-Crofts 1967; pp. 4-44.

[47] Elaad E, Ben-Shakhar G. Effects of mental countermeasures on psychophysiological detection in the guilty knowledge test. Int J Psychophysiol 1991; 11(2): 99-108.

[48] Elaad E, Ben-Shakhar G. Finger pulse waveform length in the detection of concealed information. Int J Psychophysiol 2006; 61(2): 226-34

[49] von Euler C. Brainstem mechanisms for generation and control of breathing pattern. In: Cherniack NS, Widdicombe JG, Eds. Handbook of Physiology Washington, D.C.: American Physiology Society 1986; pp. 1-67.

[50] Heywood P, Murphy K, Corfield DR, et al. Control of breathing in man insights from the 'locked-in' syndrome. Resp Physiol 1996; 106(1): 13-20.

[51] Ben-Shakhar G, Dolev K. Psychophysiological detection through the guilty knowledge technique: effects of mental countermeasures. J Appl Psychol 1996; 81(3): 273-81.

[52] Akre H, Skatvedt O. Advantages of measuring air flow in the pharynx with internal thermistors. Eur Arch Oto-Rhino-L 2000 257(5): 251-55

[53] Johansen K, Burggren WW, Eds. Skin arteriovenous anastomoses, their control and role in thermoregulation:
Cardiovascular shunts, Alfred Benzon Symposium 21; 1985, Musnksgaard, Copenhagen, 433-48.

[54] Elam M, Wallin BG. Skin blood flow responses to mental stress in man depend on body temperature. Acta Physiol Scand 1987; 129: 489-97.

[55] Kistler A, Mariauzouls C, von Berlepsch K. Fingertip temperature as an indicator for sympathetic responses. Int J Psychophysiol 1998; 29(1): 35-41.

[56] Krogstad A, Elam M, Karlsson T, et al. Arteriovenous anastomoses and the thermoregulatory shift between cutaneous vasoconstrictor and vasodilator reflexes. J Autonom Nerv Syst 1995; 53(2-3): 215-22.

[57] Pollina DA, Dollins AB, Senter SM, et al. Facial skin surface temperature changes during a "Concealed Information" test. Ann Biomed Eng 2006; 34(7): 1182-9.

[58] Bleecker ER, Engel BT. Learned control of ventricular rate in patients with atrial fibrillation. Psychosom Med 1973; 35(2): 161-75.

[59] Nikooman P, Engle BT, Vanasin B, et al. Biofeedback control of sphincter reflexes to treat fecal incontinence. Gastroenterology 1973; 64(4): 778.

[60] Birbaumer N, Flor H. Applied psychophysiology and learned physiological regulation. Appl Psychophysiol Biofeedback 1999; 24(1): 35-7.

61] Flor H, Birbaumer N. Comparison of the efficacy of electromyographic biofeedback, cognitive-behavioral therapy, and conservative medical interventions in the treatment of chronic musculoskeletal pain. J Consult Clin Psych 1993; 61(4): 653-8.

[62] Engel BT. Clinical biofeedback: A behavioral analysis. Neurosci Biobehav Rev 1981; 5(3): 397-400.

[63] Khen-Dunlop N, Van Egroo A, Bouteiller C, et al. Biofeedback therapy in the treatment of bladder overactivity, vesico-ureteral reflux and urinary tract infection. J Pediatr Urol 2006; 2(5): 424-9.

[64] Middaugh S, Haythornthwaite JA, Thompson B, et al. The Raynaud's treatment study: Biofeedback protocols and acquisition of temperature biofeedback skills. Appl Psychophysiol Biofeedback 2001; 26(4): 251-78

[65] Freedman RR, Ianni P. Self-control of digital temperature: Physiological factors and transfer effects. Psychophysiology 1983; 20: 682-9.

[66] Grabert JC, Bregman NJ, McAllister HA. Skin temperature regulation: the effects of suggestion and feedback. Int J Neurosci 1980; 10: 217-21.

[67] Keefe F, Gardner E. Learned control of skin temperature: Effects of short and long-term biofeedback training. Behav Ther 1979; 10: 202 10 .

[68] Kristt D, Engel B. Learned control of blood pressure in patients with high blood pressure. Circulation 1975; 51(2): 370-8.

[69] Kranitz LJD, Lehrer P. Biofeedback applications in the treatment of cardiovascular diseases. Cardiol Rev 2004; 12(3): 177-81.

[70] Aivazyan TA, Zaitsev VP, Yurenev AP. Autogenic training in the treatment and secondary prevention of essential hypertension: fiveyear follow-up. Health Psychol 1988; 7: 201-8.

[71] Patel CH. Yoga and biofeedback in the management of hypertension. Lancet 1973; 2: 93-5.

[72] Yucha CB, Clark L, Smith M, et al. The effect of biofeedback in hypertension. Appl Nurs Res 2001; 14(1): 29-35.

[73] Linden W. The efficacy of behavioral treatments for hypertension. Appl Psychophysiol Biofeedback 2006; 31(1): 51-63.

[74] Tsai PS, Chang NC, Chang WY, et al. Blood pressure biofeedback exerts intermediate-term effects on blood pressure and pressure reactivity in individuals with mild hypertension: a randomized controlled study. J Alter Complem Med 2007; 13(5): 547-54.

[75] Nestoriuc Y, Martin A. Efficacy of biofeedback for migraine: A meta-analysis. Pain 2007; 128(1-2): 111-27.

[76] Lykken DT. A Tremor in the Blood. New York, USA: McGraw-Hill 1981

[77] Ben-Shakhar G, Furedy JJ. Theories and applications in the detection of deception: A psychophysiological and international perspective. New York, USA: Springer-Verlag 1990.

[78] Elaad E. Detection of guilty knowledge in real-life criminal investigations. J Appl Psychol 1990; 75: 521-9.

[79] Corteen RS, Wood B. Autonomic responses to shock-associated words in an unattended channel. J Exp Psychol 1977; 94: 308-13.

[80] Davidson PO. Validity of the guilty knowledge technique: the effects of motivation. J Applied Psychol 1968; 52: 62-5. 
[81] Giesen M, Rollison MA. Guilty knowledge versus innocent associations: effects of trait anxiety and stimulus context on skin conductance. Psychophysiology 1988; 25: 683-8.

[82] Godert HW, Rill HG, Vossel G. Psychophysiological differenttiation of deception: the effects of electrodermal lability and mode of responding on skin conductance and heart rate. Int $\mathrm{J}$ Psychophysiol 2001; 40: 61-75.

[83] Lynn R. Attention, arousal and the orientation reaction. Oxford, England: Pergamon Press 1966.

[84] Timm HW. Analyzing deception from respiration patterns. J Police Sci Admin 1982; 10: 47-51.

[85] Kircher J, Raskin DC. Human versus computerized evaluation of polygraph data in a laboratory setting. J Appl Psychol 1988; 73: 291-302.

[86] Timm HW. Effect of altered outcome expectancies stemming from placebo and feedback treatments on the validity of the guilty knowledge technique. J Appl Psychol 1982; 67: 391-400.

[87] Ballantyne JR, Deva GS. Yoga sutras of Panjali Delhi. Parimal 1990; 10: 48-87.

[88] Maharishi MY. Maharishi Mahesh Yogi on the Bhagavad-Git: A new translation and commentary, Chapters 1-6. Baltimore, USA: Penguin Books 1969.

[89] Travis F. Autonomic and EEG patterns distinguish transcending from other experiences during transcendental meditation practice. Int J Psychophysiol 2001; 42(1): 1-9.

[90] Kaushik RM, Kaushik R, Mahajan SK, et al. Effects of mental relaxation and slow breathing in essential hypertension. Complement Ther Med 2006; 14(2): 120-6.

[91] Allen K, Blascovich J, Tomaka J, et al. The presence of human friends and pet dogs as moderators of autonomic reactivity to stress in women. J Perso Soc Psychol 1991; 61: 582-9.

[92] Katz L, Epstein S. Constructive thinking and coping with laboratory-induced stress. J Perso Soc Psychol 1991; 61: 789-800.

[93] Tomaka J, Blascovich J, Swart L. Effects of vocalization on cardio-vascular and electrodermal responses during mental arithmetic. Int J Psychophysiol 1994; 18(1): 23-33.

[94] Decety J, Jeannerod M, Germain M, et al. Vegetative response during imagined movement is proportional to mental effort. Behav Brain Res 1991; 42: 1-5.
[95] Bolliet O, Collet C, Dittmar A. Autonomic nervous system activity during actual and mentally simulated preparation for Movement. Appl Psychophysiol Biofeedback 2005; 30(1): 11-20.

[96] Deschaumes-Molinaro C, Dittmar A, Vernet-Maury E. Autonomic nervous system response patterns correlate with mental imagery. Physiol Behav 1992; 51(5): 1021-7.

[97] Birbaumer N. Brain-computer-interface research: Coming of age. Clin Neurophysiol 2006; 117(3): 479-83.

[98] Ming C, Xiaorong G, Shangkai G, et al. Design and implementation of a brain-computer interface with high transfer rates. IEEE T BioMed Eng 2002; 49(10): 1181-6.

[99] Doherty EP, Cockton G, Bloor C, et al. Yes/No or Maybe--further evaluation of an interface for brain-injured individuals. Interact Comput 2002; 14(4): 341-58.

[100] Spinney L. Hear My Voice. New Scientist, Feburary 22, 2003; pp. 36.

[101] Thornett CEE. Designing special switches and control systems for multiply handicapped young people - a problem-led approach. J Med Eng Technol 1990; 14: 717-21.

[102] Scherer MJ. The change in emphasis from people to person: introduction to the special issue on Assistive Technology. Disabil Rehabil 2002; 24(1-3): 1-4.

[103] Krishnamurthy N, Ahamed SM, Vengadesh GS, et al. Influence of respiration on human sympathetic skin response. Indian $\mathrm{J}$ physiol pharmacol 1996; 40(4): 350-4.

[104] Turpin G, Schaefer F, Boucsein W. Effects of stimulus intensity, risetime, and duration on autonomic and behavioral responding: Implications for the differentiation of orienting, startle, and defense responses. Psychophysiology 1999; 36(4): 453-63.

[105] Patterson J, Grabois M. Locked-in syndrome: a review of 139 cases. Stroke 1986; 17(4): 758-64.

[106] Cariga P. Organisation of the sympathetic skin response in spinal cord injury. J Neurol Neurosur Ps 2002; 72(3): 356-60.

[107] Alavian-Ghavanini MR, Jazayeri-Shooshtari SM, Setoudenia S, et al. Value of sympathetic skin response in multiple sclerosis. Electromyo Clin Neuro 1999; 39(8): 455-9.

[108] Dettmers C, Fatepour D, Faust H, et al. Sympathetic skin response abnormalities in amyotrophic lateral sclerosis. Muscle Nerve 1994; 17(8): 957-8.

\footnotetext{
Received: April 22, 2008

(C) Blain et al.; Licensee Bentham Open.
}

Revised: July 07, 2008

Accepted: July 14, 2008

This is an open access article licensed under the terms of the Creative Commons Attribution Non-Commercial License (http://creativecommons.org/licenses/by$\mathrm{nc} / 3.0 /$ ), which permits unrestricted, non-commercial use, distribution and reproduction in any medium, provided the work is properly cited. 